\title{
Dyslexia and Auditory Processing Disorder: A Neurological Chicken or Egg Debate?
}

\author{
Isabella Summe ${ }^{1}$ and J. R. McCoy ${ }^{2}$ \\ ${ }^{1}$ Sacred Heart Schools, Atherton, Atherton, CA, USA \\ ${ }^{2}$ Veterans Affairs Hot Springs, AR, USA and UAMS, Little Rock, AR, USA \\ ABSTRACT
}

An estimated 10 million students in the United States have dyslexia and of those students, 2.5 million are estimated to have auditory processing disorder. There is an ongoing debate on whether auditory processing disorder causes or contributes to dyslexia or the disorders are just co-morbid. This paper examines dyslexia and auditory processing disorder and then considers current research on the nature of the relationship between the two disorders.

\section{Introduction}

An estimated one in five individuals in the United States has a language-based learning disability (Ferrer et al., 2015, p. 1121; Yale Center for Dyslexia). Dyslexia, commonly described as an unexpected and chronic difficulty in reading caused by phonological processing challenges, is the most prevalent of the language-based learning disabilities (Lyon, Shaywitz \& Shaywitz, 2003, p. 1). The cause of dyslexia is not known. However, advancements in medical imaging have highlighted structural differences between a dyslexic brain and a neurotypical brain. An emerging area of research is the relationship between dyslexia and auditory processing disorder (APD), a specific deficit in auditory processing along the central nervous system. The National Institutes of Health, citing Iliadou's research, found that $43 \%$ of children referred for learning disabilities have APD, with $25 \%$ of children tested for learning disabilities having co-existing dyslexia and APD (Iliadou V. et al., 2009, p. 1029; National Institutes of Health). A debate as to whether dyslexia is caused by APD or is the result of atypical brain-based structures has begun to take shape. This paper examines dyslexia and APD and considers current research on the nature of the relationship between the two disorders. While this research is in its infancy, its ultimate findings will have a profound effect on the significant number of individuals diagnosed with one or both disorders.

\section{Background}

The auditory system is crucial for the development of language. The brain's inability to rapidly process auditory input is a hallmark of language disorders like dyslexia (Chevalier et al., 2015, p. 1-2). There is evidence that many dyslexics have auditory perceptual deficits (Halliday et al., 2017). At present, however, there is no research consensus on whether auditory processing deficits cause dyslexia or whether dyslexia's phonological processing challenges diminish auditory processing. This seems to be a classic chicken or egg problem: does one disorder cause the other? Alternatively, do the disorders simply co-exist with one another or does one disorder create enhanced risk factors for the development of another? 


\section{Dyslexia}

Dyslexia was identified in 1887 by a German neurologist, Adolph Kussmaul, who labeled it "word blindness" given his observation that the affected individual had the intellectual and visual capacity to read, but yet could not (Rehnberg \& Walters, 2017, p. 72). In the same year, a German ophthalmologist, Rudolf Berlin, first used the word "dyslexia" to describe six adult patients who lost their ability to read following the development of a lesion in the brain (Shaywitz \& Shaywitz, 2020, p. 16). In 1896, W. Pringle Morgan in England published an article in the British Medical Journal describing the word blindness of his fourteen-year old patient (Morgan, 1896, p. 1378), which unleashed a flood of other ophthalmologists and other physicians who noticed similar challenges in their patient populations. Today, the Diagnostic and Statistical Manual of Mental Disorders-5 classifies dyslexia as a form of neurodevelopmental disorder which impedes the ability to learn or use specific academic skills (e.g., reading, writing or arithmetic), noting that such difficulties are "unexpected" (DSM-5, 2013). U.S. legislation defines dyslexia as: "an unexpected difficulty in reading for an individual who has the intelligence to be a much better reader, most commonly caused by a difficulty in the phonological processing (the appreciation of the individual sounds of spoken language), which affects the ability of an individual to speak, read, and spell." (First Step Act 2018). Sally Shaywitz, a long-time dyslexic researcher and advocate who was instrumental in the development of the First Step Act, states that dyslexia is "characterized by the frequent co-occurrence of high IQ and surprisingly slow reading" (Shaywitz \& Shaywitz, 2020, p. 89-90). As a neurotypical child develops phonemic awareness, she learns that specific sounds connect to letters, which can be combined into words. In a dyslexic child, there is an impairment in the child's phonemic awareness - a "glitch within the language system" (Shaywitz \& Shaywitz, 2020, p. 51), resulting in difficulty in breaking apart sounds.

While the acquisition of language is arguably innate (Pinker, 2007, p. 5), the application of language to reading is not. In fact, while language has been represented in humans for an estimated million years (Shaywitz \& Shaywitz, 2020, p. 45), reading and writing are more recent developments in the last five thousand years and skills that must be taught. Language is composed of building blocks of sound called phonemes. The English language has forty-four phonemes. (The Reading Well). These forty-four phonemes can be combined in a myriad of ways to create words. Any difficulty in breaking apart, segmenting, and manipulating sound can create challenges in reading and speaking.

Consider the neurological complexity of reading the word "cat". The word "cat" has three distinct and separate units of sound - one for each letter: $k$, aaaa, and $t$ (Shaywitz \& Shaywitz, 2020, p. 49). When a dyslexic sees the word "cat", she must break down the sound associated with each letter, organize the sounds in the proper sequence, and then blend the sounds together to form one word - cat. Sometimes, a dyslexic retrieves the wrong phoneme, mixing up one letter and its corresponding sound for another, or she orders the phonemes in the wrong sequence. While typical readers scale to recognize the word "cat" when it appears in text, relying on their working memory, a dyslexic's phonemes and working memory skills are less well-developed so rapid word retrieval and sight recognition of repetitive words is more difficult. A dyslexic's phonological weakness does not just affect reading. It can also interfere with expressive speech. Often, dyslexics know the "right" word to say but invert or substitute letters, causing the "wrong" word to be said. The phonologic module "automatically assembles the phonemes into words for the speaker and disassembles the spoken word back into its underlying phonemes for the listener" (Shaywitz, 2003, p. 45; Shaywitz \& Shaywitz, 2020, p. 55-56). Consequently, a dyslexic has difficulty with phonemic awareness, which is a critical skill in identifying and manipulating sounds. For example, a young dyslexic would struggle with being asked to substitute "m" for "c" to get "mat" rather than "cat." Transforming letters on a page into their phonetic sounds is neurological magic and happens automatically for neurotypical children, but dyslexic children never experience this automaticity. Shaywitz calls a dyslexic's viewpoint an "amphorous blur" - an inability to discern the segmentation of each letter's sound (Shaywitz, 2003, p. 43-44).

A dyslexic's phonological weakness can be traced to specific structures in the brain that do not activate in the same way as a neurotypical individual. The brain has two hemispheres - the left and the right. At a general level, the left hemisphere of the brain is responsible for language, speech, comprehension, arithmetic, and writing. The right 
hemisphere plays a large part in interpreting visual information. The right hemisphere controls creativity, spatial ability, artistic skill, and musical skill. Each hemisphere of the brain has four sections or lobes: frontal, parietal, temporal, and occipital. The two hemispheres are connected by the corpus callosum, a thick bundle of nerve fibers that reside beneath the cerebral cortex.

There are three neural pathways or regions of the brain that are used for reading: (i) the parieto-temporal pathway; (ii) Broca's area; and (iii) the occipito-temporal pathway (Shaywitz \& Shaywitz, 2020, p. 74). (Fig. 1) The parieto-temporal pathway and Broca's area are most activated in neurotypical beginning readers, whereas the occipitotemporal pathway is relied upon by experienced readers. The parieto-temporal pathway, located in the middle of the brain, is where readers link letters to their corresponding sounds. Broca's area, located in the frontal lobe of the brain, is where the articulation of words occurs. The organization, production, and manipulation of language and speech is dependent on this region of the brain. (Ozernov-Palchik \& Gabrieli, 2018, p.16).

The occipito-temporal pathway, located at the posterior of the brain, is where the primary visual cortex resides. This neural pathway identifies words through visual perception and is referred to as the "Visual Word Form Area", given that the visual cortex identifies whole words with automaticity (Shaywitz \& Shaywitz, 2020, p. 82). After a reader analyzes a word multiple times, an exact neural model of that word - how it is spelled, how it is pronounced, and what it means - is developed. Shaywitz pointed out that it used to be thought that the neural model of words was permanently stored in the occipito-temporal system (Shaywitz, 2003, p. 79), but today it is hypothesized that the occipital-temporal region is an " "interactive" neural mode where information from multiple inputs, including phonological (sound-based), orthographic (visual), and semantic (meaning-based), are integrated and synthesized" (Shaywitz \& Shaywitz, 2020, p. 76).

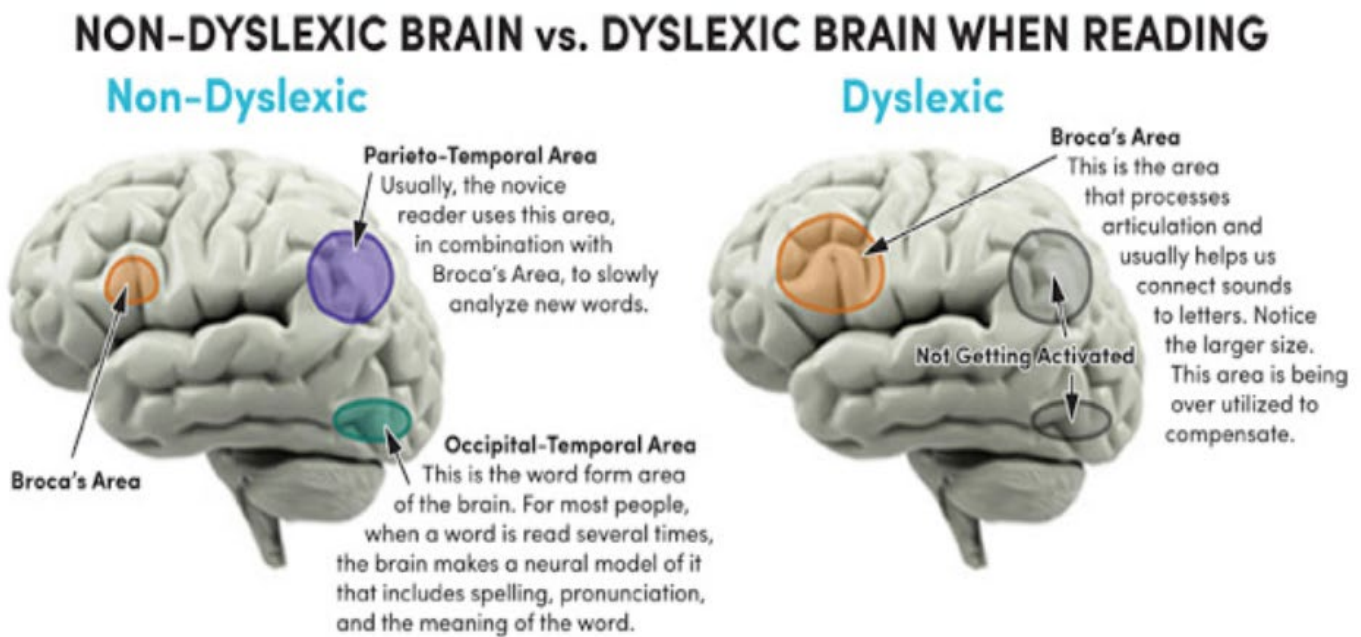

\begin{abstract}
Research in neuroscience reveals that the brain functions differently in people with dyslexio thon those without it. These structural and neural differences make it more difficult for people with dyslexia to read, spell and write. For example, in the left brain hemisphere, three dominant oreas of the brain are usually activated for reading, but in those with dyslexia, only one area of the brain is being stimulated.
\end{abstract}

Figure 1. A Comparison of Key Brain Areas in Dyslexics and Non-Dyslexics. Accessed at http://cognitive.com.sg/understanding-dyslexia/.

For a dyslexic, the neural pathways necessary for phonological analysis are mis-wired, resulting in a phonologic impairment that interferes with spoken and written language. The lack of electrical activation of the parieto-temporal pathway results in a dyslexic reader's struggle to link letters to sounds. Further, the diminished activity in the occipitotemporal pathway contributes to a dyslexic reader's challenge with automatic word identification. This degradation 
of automaticity in retrieving words negatively impacts reading skill. A dyslexic compensates for the weakness in these two neural pathways by overusing Broca's area (Shaywitz \& Shaywitz, 2020, p. 80). Even though a dyslexic can use Broca's area and other auxiliary systems to read, it is a slow process given that the storage and retrieval of neural models of words is diminished.

It is estimated that $50 \%$ of children with a "first degree" relative like a parent with a dyslexia diagnosis will also be diagnosed with dyslexia (Ozernov-Palchik \& Gabrieli, 2018, p. 17). It should be noted that there is research on the identification of specific genes that may contribute or cause dyslexia, as well as examination of the volume of white and grey matter in one's brain and its possible relationship to dyslexia (Kraus \& White-Schwoch, 2013; Chevalier et al., 2015, p.1-2). While those areas are outside of the scope of this paper, it is hoped that this research will lead to greater insights in the identification and intervention strategies most effective for dyslexics.

\section{Auditory Processing Disorder}

Auditory Processing Disorder (APD) is a learning disorder that affects how the brain processes what a person hears. APD has been referred to as the "auditory equivalent of dyslexia" (Bellis, 2002, p. i). APD is not a hearing-based or ear-based auditory problem, but rather a deficit in the central auditory system - the portion of the central nervous system that runs from the ear to the brainstem. In other words, APD is a deficiency in how an individual's central nervous system uses or processes auditory information. It is estimated that 2.5 million students, about $5 \%$ of the student population, have APD (Hearing Health Foundation).

The central auditory system is responsible for the recognition, localization (direction of the source of a sound), and discrimination of sound (isolation of sounds from one another). The cochlea, commonly referred to as the inner ear, are mirror-shaped and filled with fluid, and are deeply embedded in the skull's petrous bone, one of the hardest and most dense bones in the human body. The hardness of the bone is important because it prevents the fluid from being absorbed into the bone.

All acoustic information is relayed from the outer ear to the cochlea. The cochlea then transmit the acoustic information to the auditory or cochlear nerves, and the cochlear nerves then transmit the acoustic information to the brainstem. Inside the cochlear nerves, there are an estimated 28,500 nerve fibers (Moore, 1992, p. 26). These nerve fibers are divided into two types: (i) type 1 myelinated fibers that connect to inner hair cells in the cochlear nuclear complex, which comprise $95 \%$ of the auditory nerve fibers; and (ii) type 2 unmyelinated fibers that connect to outer hair cells, which comprise 5\% of the auditory nerve fibers (Chermak \& Musiek, 1997, p.28). Myelination refers to the fatty sheath that surrounds neurons. Myelination enhances efficient electrical transmission of acoustic information. Several studies point to the possible correlation of the amount of white matter, which is found in the deeper tissues of the brain, with nerve fibers covered in myelin, and reading fluency and accuracy (Chevalier et al., 2015, p. 2; Lebel et al., 2019), noting that more research is needed.

After an acoustic signal travels from the cochlear nucleus to the brainstem, it continues its journey to the auditory cortex (Figure 2). The auditory cortex is located in the temporal lobe of the brain, which is mainly responsible for understanding language (Chermak \& Musiek, 1997, p. 45). The brain can then synthesize the information and bring it to where it needs to go. Important skills needed to process this acoustic information are phonemic awareness and word decoding skills. The listener must be able to identify the spoken phonemes to be able to understand the information. Another important factor in processing this information is the interpretation of the language, which takes

place in Wernicke's area, which is close to the auditory cortex. Wernicke's area is responsible for comprehending the speech sounds, specifically receptive language (Shaywitz, 2003, p. 66). 


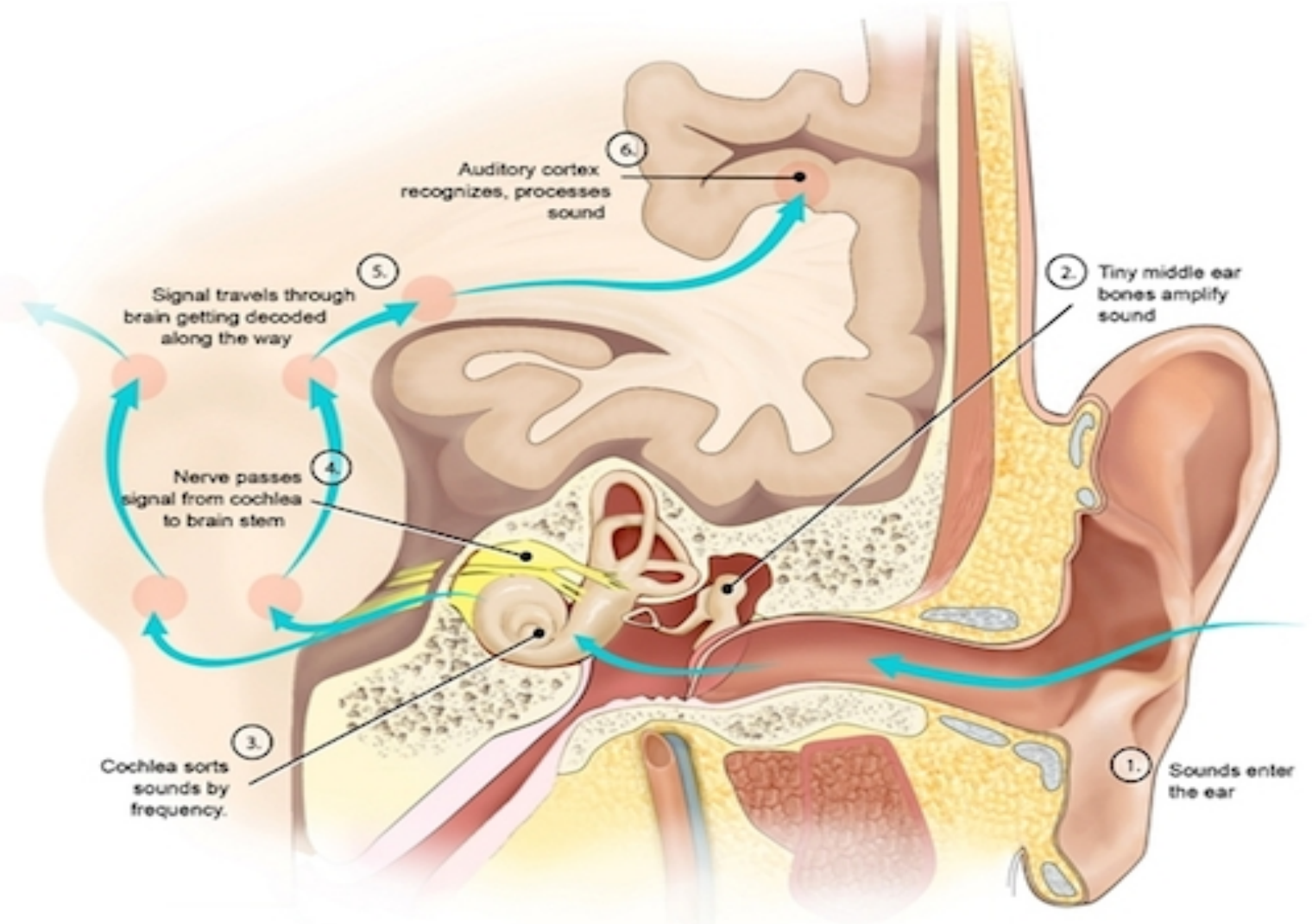

Figure 2. Auditory Processing System. Accessed at https://study.com/academy/lesson/what-is-auditory-processingdisorder-symptoms-treatment.html.

If the central auditory system is not functioning correctly, information does not make its way to the auditory cortex intact. Therefore, individuals with APD can often completely lose sounds because those sounds do not make it to the auditory cortex in the brain for processing. Since the individual loses part of what she is hearing, it is difficult for her to comprehend all sounds and to put the words into an ordered context. This inability to make the acoustic signal travel all the way to the auditory cortex is what makes auditory processing slower or more interrupted.

Research seems to conclude that APD is not a single disorder, but rather a collection of functional deficits (Chermak \& Musiek, 1997, p. 1). There are different types or presentations of APD, including deficits in decoding sounds and the formation of words, the integration of sounds, poor prosody (patterns of rhythm and intonation in speech), associative deficiencies, and output-organization (organization and then production of speech). What is clear is that individuals diagnosed with APD experience challenges in isolating and hearing sound in environments where there is competing noise. Auditory discrimination and pattern recognition is therefore more challenging, with performance or accuracy declining the more there are competing noises in the individual's environment. Understanding how an individual's auditory processing challenges relates to the development of language based processing disorders has yet to be fully explored.

\section{Does Dyslexia or APD Come First?}

Considerable research supports the view that many dyslexics also have auditory processing deficits (Halliday et al., 2017, p. 140). It is estimated that $10 \%$ of the general population has an abnormal range for learning skills, of which $37 \%$ of that population will have dyslexia and $46 \%$ will be co-morbid with APD (Cunha et al., 2019, p. 120, citing 
Dawes \& Bishop, 2010). Recent research found that forty percent of children with dyslexia have another diagnosed neurological disorder (Snowling et al., 2020, p. 505). Those other neurological disorders, besides APD, include attention deficit disorder (ADHD), dysgraphia, dyscalculia, developmental coordination disorder, and others (Sakellariou, 2016, p. D-27).

How these auditory processing deficits are related to dyslexia is unknown - is it the cause of dyslexia or a contributing risk factor? Current research cannot yet answer this question definitively, but experts agree that auditory processing deficits are, at a minimum, an early warning sign of dyslexia. For example, the American Speech-Language-Hearing Association (ASHA) has stated that "auditory perceptual abilities influence language development particularly the pre-literacy skills." Sakellariou argues that "some research indicates that auditory processing skills could be the primary shortfall in dyslexia" (Sakellariou, 2016, p. D-28). When an individual has APD, it makes it harder to "detect and process speech patterns" (Sakellariou, 2016, p. D-28). APD may not cause dyslexia, but it seems to make a dyslexic's ability to clearly discern and segment sounds more challenging. Starting with an evaluation of an individual's auditory processing is useful because any deficiency can be diagnosed at an earlier age than dyslexia. There is evidence that phonological deficits are present in preschool children, well before reading instruction begins (Snowling et al., 2020, p. 504).

There are two principal challenges in understanding the relationship among APD and dyslexia. First, there is not consensus definition of dyslexia. The lack of an agreed-upon definition of dyslexia means that there is inconsistent criteria for diagnosis. If there is inconsistent criteria for a diagnosis of dyslexia, then the role of any auditory processing deficit may go undetected. Second, the lack of a holistic approach to the evaluation, identification, and intervention strategies applied to co-occurring APD and dyslexia is also problematic. Any significant delay in identifying and intervening earlier in children's lives, when neural circuitry may be more receptive to intervention, creates learning challenges down the road.

\section{Expansion of Dyslexia's Diagnosis}

With respect to the first challenge, the most common definition of dyslexia as a "specific" learning disability means that the individual's intellectual skills are not deficient, and thus cannot serve as the explanation for the dyslexia (Snowling et al., 2020, p. 502). The cause must be something "specific" neurologically. Over time, inconsistent definitions of dyslexia have served to encompass within the diagnosis of dyslexia a wide range of other learning disabilities, thereby masking appropriate identification and targeted intervention strategies not only for dyslexia, but for the other learning disabilities that are present.

The broadening of the definition of dyslexia results from an increase in the prevalence of dyslexia in schoolaged children, which could emanate from greater awareness or overly broad diagnoses (Snowling et al., 2020, p. 506). The typical phonological awareness challenges that a dyslexic has does not necessarily result from or lead to a diagnosis of APD, but blurring the lines between dyslexia and other learning disorders, such as ADHD, working memory challenges, or executive functioning or organizational struggles, may obscure, rather than illuminate, any connection between dyslexia and APD. Because phonological deficits are tied to dyslexia, it overlooks the fact that phonological skills themselves are multi-dimensional as well. If early language problems result in downstream phonological difficulties, then the additional challenges relating to organization, comprehension, and word de-coding are likely to be materially affected. The development of vocabulary, syntax (arrangement of words to form sentences), and speech, may also play a role. Another risk of the expansion of the definition of dyslexia is that it may include individuals with more serious language difficulties that cause chronic difficulties in expressive and/or receptive language (Snowling et al., 2020, p. 505). It is estimated that of the dyslexic children population, $40 \%$ had significant language impairment (Snowling et al., 2020, p. 505). The development of consensus criteria to assign a dyslexia diagnosis is missing. Without clarity of diagnostic criteria, individuals suffer from mis-identification or under-identification of one or more neurologically-based learning disorders. 


\section{A Holistic Approach to Evaluating, Identifying, and Developing Intervention Strategies}

The second challenge is to develop a holistic approach to evaluating, identifying, and developing intervention strategies for dyslexia and/or APD. Today, there is not comprehensive agreement on the diagnostic standards for either. The way an individual is referred for evaluation may impact the interventions prescribed. Typically, school-aged children are referred for a dyslexia evaluation by a clinical neuropsychologist after chronic or persistent difficulty with reading is evidenced in school. That means a child may be in second or third grade before an evaluation for dyslexia is completed. Children referred for APD evaluation are typically in first or second grade and evaluated by audiologists. Standard procedures for diagnosing APD usually include audiological tests, auditory brainstem tests, and auditory processing tests (ASHA). These tests may be influenced by cognitive factors, such as sustained auditory attention. The association between attention and auditory processing suggest that APD diagnostic criteria should be reconsidered, as they do not currently routinely include cognitive diagnostic measures.

Because of specialization by professionals evaluating for dyslexia, APD or other learning disabilities, comorbid diagnoses may be overlooked, even though there may be neurodevelopmental similarities. This impacts the creation of successful intervention strategies. For example, there is significant co-morbidity of APD and ADHD, and this has led to confusion in identification and specific interventions (Stavrinos et al., 2018, p. 1). Best practice clinical guidelines for the treatment of ADHD include a combination of medication along with behavioral interventions (Centers for Disease Control and Prevention). Medication is not, however, a recommended treatment for children with APD. Interestingly, performance on auditory tasks such as discrimination of sound in noise, temporal processing, and auditory attention improves in children following a course of Ritalin or its equivalent stimulant that is prescribed to children with ADHD (Centers for Disease Control and Prevention).

Different viewpoints among audiologists, clinical neuropsychologists, and other professionals such as speech language pathologists, exist for a number of reasons, including the heterogeneity of symptoms, variations in the definition of APD and dyslexia and other learning disorders, the lack of a reference standard for diagnosis, the relationship between auditory perceptual deficits and language disorders, and the particular treatment approach(es) that follow from the diagnosis. A cross-functional approach to evaluating children identified with any one of dyslexia, APD or even ADHD, should result in earlier intervention to address weaknesses in neural systems. It is also likely that continued advancements in technology will increasingly assist in diagnosis. Functional magnetic resonance imaging and electrophysiology have contributed to an enhanced understanding of the brain's structural aspects and its relationship with language-based learning disabilities. Cerebral maturation and cognitive development are interdependent processes, and stages of cognitive development have associated stages of myelination and dendritic branching throughout childhood and into adolescence (Chevalier et al., 2015, p.2). While it is unlikely that brain imaging will become standard screening, the early discernment of under-performing areas of the brain would, without doubt, aid intervention strategies, particularly for dyslexia, in an effort to rewire the brain's circuitry.

\section{Conclusion}

An estimated 10 million school-aged children have dyslexia (United States Census Bureau; International Dyslexia Association; Shaywitz \& Shaywitz, 2020, p. 7, 29). An accurate assessment of how many of these dyslexic children also have APD is not known but estimates range as high as 25 percent (Iliadou V. et al., 2009, p. 1029; National Institutes of Health). Many dyslexics are not ever evaluated for APD. Hence, it is not known whether APD is a contributing risk factor or a cause of dyslexia's language-based neurological deficits.

Embracing the multi-dimensional nature of auditory processing and dyslexia is needed to address the learning challenges faced by a significant percentage of our fellow students. Societal stigmas associated with a range of learning disorders have lessened and educational environments are slowly learning to be more attuned to possible early 
diagnosis and intervention. Developing holistic evaluation and intervention efforts will lead to greater human potential outcomes for these individuals.

\section{Acknowledgments}

I would like to thank my advisor Dr. McCoy for helping me with this project. Additionally, I would like to thank Dr. Caryn Kovar who shared with me on January 26, 2021, as I was finalizing this paper, that I was diagnosed with dyslexia. I would also like to thank my younger brother, Christian Summe, who has dyslexia. Upon learning that I, too, had dyslexia, he beamed "Welcome to the club!".

\section{References}

Alpiner, J.G. \& McCarthy, P.A. (1992). Rehabilitative Audiology: Children and Adults (2 ${ }^{\text {nd }}$ ed). Baltimore: Williams $\&$ Wilkins.

American Psychiatric Association. (2013). Diagnostic and Statistical Manual of Mental Disorders-5 (5 ${ }^{\text {th }}$ ed.). Washington, D.C.: Author.

American Speech-Language-Hearing Association. Central Auditory Processing Disorder. Retrieved on October 16, 2020, from https://www.asha.org/Practice-Portal/Clinical-Topics/Central-Auditory-Processing-Disorder/.

Baran, J.A., Jones, R.O., Musiek, F.E. \& Shinn, J.B. (2021). Disorders of the Auditory System. (2 ${ }^{\text {nd }}$ ed.) San Diego: Plural Publishing Inc.

Bauman, K. \& Cranney, S. School Enrollment in the United States: 2018. United States Census Bureau P20-584. Retrieved January 29, 2021, from https://www.census.gov/content/dam/Census/library/publications/2020/demo/p20-584.pdf.

Benasich, A. A., Thomas, J. J., Choudhury, N., \& Leppänen, P. H. (2002). The importance of rapid auditory processing abilities to early language development: evidence from converging methodologies. Developmental Psychobiology, 40(3), 278-292. https://doi.org/10.1002/dev.10032.

Centers for Disease Control and Prevention. Treatment of ADHD. Retrieved October 17, 2020, from https://www.cdc.gov/ncbddd/adhd/treatment.html.

Chermak, G.D. \& Musiek, F.E. (1997). Central Auditory Processing Disorders: New Perspectives. San Diego: Singular Publishing Group. Inc.

Chevalier, N., Kurth, S., Doucette, M. R., Wiseheart, M., Deoni, S. C., Dean, D. C., O’Muircheartaigh, J., Blackwell, K. A., Munakata, Y., \& LeBourgeois, M. K. (2015). Myelination Is Associated with Processing Speed in Early Childhood: Preliminary Insights. PLOS One, 10(10), e013989, 1-14. https://doi.org/10.1371/journal.pone.0139897.

Cunha, P., Monteiro de Castro Silva, I., Rabelo Neiva, E., \& Tristão, R.M. (2019). Auditory Processing Disorder Evaluations and Cognitive Profiles of Children with Specific Learning Disorder. Clinical Neurophysiology Practice, 4, 119-127. 
Dawes, P. \& Bishop, D.V.M. (2010). Psychometric Profile of Children with Auditory Processing Disorder and Children with Dyslexia. Archives of Disease in Childhood, 95, 432-436. https://doi.org/10.1136/adc.2009.170118.

Ferrer, E., Holahan, J.M., Marchione, K., Michaels, R., \& Shaywitz, B. (2015). Achievement Gap in Reading is Present as Early as First Grade and Persists through Adolescence. Journal of Pediatrics, 167(5), 1121-25. https://doi.org/10.1016/j.jpeds.2015.07.045.

First Step Act: 132 Stat. 5194 Public Law No. 115-391. December 21, 2018.

Galaburda, A.M., Sherman, G.F., Rosen, G.D., Aboitz, F., \& Geschwind, N. (1985). Developmental Dyslexia: Four Consecutive Patients with Cortical Abnormalities. Annals of Neurology, 18(2): 222-233. https://doi.10.1002/ana.410180210.

Halliday, L.F., Tuomainen, O. \& Rosen, S. (2017). Auditory Processing Deficits are Sometimes Necessary and Sometimes Sufficient for Language Difficulties in Children: Evidence from Mild to Moderate Sensorineural Hearing Loss. Cognition, 166, 139-151. https://doi.org/10.1016/j.cognition.2017.04.014.

Hearing Health Foundation. Retrieved on January 15, 2021, from https://hearinghealthfoundation.org/apd-demographics\#: :text=In\%20the \%20U.S. $\% 2$ C $\% 20$ it $\% 20$ is, $2.5 \% 20$ million $\% 20$ children $\% 2$ C $\% 20$ have $\% 20$ APD.

Holahan, J.M., Kenney, B., Shaywitz, B., \& Shaywitz, S. (2020). The Yale Outcome Study: Outcomes for Graduates with and without Dyslexia. The Journal of Pediatric Neuropsychology, 6, 189-97.

https://doi.org/10.1007/s40817-020-00094-3.

Holland, J.L. (2014). Train the Brain to Hear: Understanding and Treating Auditory Processing Disorder and Other Learning Disabilities ( $2^{\text {nd }}$ ed.). Boca Raton: Universal-Publishers.

Hull, R.H. (1992). Aural Rehabilitation (2 ${ }^{\text {nd }}$ ed.). San Diego: Singular Publishing Group, Inc.

Iliadou V., Bamiou D.E., Kaprinis S., Kandylis D., \& Kaprinis G. (2009). Auditory Processing Disorders in Children Suspected of Learning Disabilities - A Need for Screening? International Journal of Pediatric Otorhinolaryngology. 73(7), 1029-34. https://doi:10.1016/j.ijporl.2009.04.004.

International Dyslexia Association. Retrieved on December 27, 2020, from https://dyslexiaida.org/dyslexia-basics/.

Kraus, N. \& White-Schwoch, T. (2013). Physiologic Discrimination of Stop Consonants Relates to Phonological Skills in Pre-Readers: A Biomarker for Subsequent Reading Ability? Frontiers in Human Neuroscience. https://doi.org/10.3389/fnhum.2013.00899

Lebel, C., Benischek, A., Geeraert, B., Holahan, J., Shaywitz, S., Bakhshi, K., \& Shaywitz, B. (2019). Developmental Trajectories of White Matter Structure in Children with and without Reading Impairments. Developmental Cognitive Neuroscience. 36, 100633. https://doi.org/10.1016/j.dcn.2019.100633.

Lyon, G. R., Shaywitz, S., \& Shaywitz, B. A. (2003). A Definition of Dyslexia. Annals of Dyslexia, 53(1), 1-14. https://doi.org/10.1007/s11881-003-0001-9. 
Middlebrooks, J.C. (2015). Auditory System: Central Pathways. Reference Module in Biomedical Sciences. https://doi.org/10.1016/B978-0-12-801238-3.04506-2 and http://www.sciencedirect.com/science/article/pii/B9780128012383045062.

Moll, K., Snowling, M.J., \& Hulme, C. (2020). Introduction to the Special Issue of "Comorbidities Between Reading Disorders and Other Developmental Disorders.” Scientific Studies of Reading, 24(1), 1-6.

Moore, B.C.J. (1992). An Introduction to the Psychology of Hearing (3 ${ }^{\text {rd }}$ ed.) San Diego: Academic Press Inc.

Morgan, W. Pringle (1896). A Case of Congenital Word Blindness. British Medical Journal, $2,1378$.

National Institute of Neurological Disorders and Stroke. (2017). Dyslexia information page. Retrieved on November 7, 2020 from https://www. ninds.nih.gov/Disorders/All-Disorders/Dyslexia-Information-Page.

Ozernov-Palchik, O. \& Gabrieli, J.D.E. (2018). Neuroimaging, Early Identification, and Personalized Intervention for Developmental Dyslexia. Perspectives on Language and Literacy, 15-20.

Pinker, Steven. (2007). The Language Instinct: How the Mind Creates Language (2 ${ }^{\text {nd }}$ ed.). New York: Harper Perennial Modern Classics.

Rehnberg, V. \& Walters, E. (2017). The Life and Work of Adolph Kussmaul 1822-1902: 'Sword Swallowers in Modern Medicine'. Journal of the Intensive Care Society, 18(1), 71-72. https://doi.org/10.1177/1751143716676822.

Sakellariou, G.I. (2016). Dyslexia, Relevant Learning Disabilities and Biological Parameters - An Approach across Languages with Different Orthographies. A Journal in Naval Sciences and Technology. Part D: Humanities and Political Sciences, 6, D27-33.

Shaywitz, J. \& Shaywitz, S. (2020). Overcoming Dyslexia (2 ${ }^{\text {nd }}$ ed.). New York: Vintage Books.

Shaywitz, S. (2003). Overcoming Dyslexia. New York: Vintage Books.

Skoe, E. \& Kraus, N. (2010). Auditory Brain Stem Response to Complex Sounds: A Tutorial. Ear and Hearing, 31(3), 302-334.

Snowling, M.J., Hulme, C., \& Nation, K. 2020. Defining and Understanding Dyslexia: Past, Present and Future. Oxford Review of Education, 46:4, 501-513. https://doi.org/ 10.1080/03054985.2020.1765756.

Stavrinos, G., Iliadou, V.M., Edwards, L., Sirimanna, T. \& Bamiou, D. 2018. The Relationship between Types of Attention and Auditory Processing Skills: Reconsidering Auditory Processing Disorder Diagnosis. Frontiers in Psychology, 9, 1-13. https://doi.org/10.3389/fpsyg.2018.00034.

The Reading Well. https://www.dyslexia-reading-well.com/44-phonemes-in-english.html.

Tomlin, D. \& Rance, G. (2016). Maturation of the Central Auditory Nervous System in Children with Auditory Processing Disorder. Seminars in Hearing, 37(1), 74-83.

Yale Center for Dyslexia. Retrieved on December 29, 2020, from https://dyslexia.yale.edu/dyslexia/what-is-dyslexia/. 